\title{
Construction of Regional Logistics Weighted Network Model and Its Robust optimization: Evidence from China
}

\author{
Lizhao Yan, ${ }^{1}$ Yi Wen, ${ }^{1}$ Kok Lay Teo, ${ }^{2,3}$ Jian Liu $\left(D,{ }^{4}\right.$ and Fei $\mathrm{Xu}^{5}$ \\ ${ }^{1}$ College of Business, Hunan Normal University, Changsha, Hunan 410081, China \\ ${ }^{2}$ School of Mathematical Sciences, Sunway University, Subang Jaya, Selangor 47500, Malaysia \\ ${ }^{3}$ Coordinated Innovation Center for Computable Modeling in Management Science, \\ Tianjin University of Finance and Economics, Tianjin 300222, China \\ ${ }^{4}$ School of Economics and Management, Changsha University of Science and Technology, Changsha, Hunan 410004, China \\ ${ }^{5}$ Department of Mathematics, Wilfrid Laurier University, Ontario N2L 3C5, Canada
}

Correspondence should be addressed to Jian Liu; ljorg@126.com

Received 2 May 2020; Revised 24 August 2020; Accepted 2 September 2020; Published 14 September 2020

Academic Editor: Ruofeng Rao

Copyright (c) 2020 Lizhao Yan et al. This is an open access article distributed under the Creative Commons Attribution License, which permits unrestricted use, distribution, and reproduction in any medium, provided the original work is properly cited.

In this paper, we construct a regional logistics model from a macroperspective. First, based on the gravity model, the index of logistics attraction between cities is established as the weight of the model, and hence the regional logistics weighted model is constructed. Next, we use the social network analysis method to analyze its structure and make specific recommendations for the construction of logistics networks. Finally, we analyze the model's response to random attacks and deliberate attacks. From our study, it is found that when the failure nodes or edges reach a certain percentage, the regional logistics network will collapse on a large scale. Therefore, it is important to optimization the threshold of the regional logistics network. This clearly provides a new perspective for the study of the regional logistics networks.

\section{Introduction}

With the rapid development of e-commerce and the increasing close cooperation between enterprises, the logistics industry has become the focus of attention from all walks of life. On the other hand, the demand for regional logistics network is also increasing. At present, the study of regional logistics network mainly focuses on the allocation and coordination of logistics resources among enterprises, which include (i) the location of the logistics distribution center; (ii) the layout of the regional logistics network; and (iii) the emergency response capability and regional logistics network optimization, for example, the P-median problem [1] which considers the location of the logistics center [2-5] from the viewpoint of the shortest distance from all logistics nodes to the logistics center. On the other hand, the location of the logistics center problem has been studied from the perspective of covering problem [6-9] and fixed-charge facility location problem [10-13]. The layout of the regional logistics network is mainly modeled as a hub-and-spoke network, where the logistics center is the "axis" and the logistics connections with other nodes are the "spokes". Such networks are studied to achieve minimum cost or optimal transportation efficiency [14-18]. In the existing literature, the emergency response capabilities of regional logistics networks (such as extreme weather) are also being discussed. For example, Wang and Xiao [19] proposes a chain failure recovery method for supply chain network based on ant colony algorithm. Hu et al. [20] proposes a multitask model to study the impact of road congestion on the regional logistics network and develops an optimization strategy. We have noticed that the current research on regional logistics network is mainly from a microperspective, using econometric analysis method to analyze a certain aspect of the logistics industry. However, such research does not provide enough results to reflect macrocharacteristics. The complex network theory developed in recent years provides a tool for regional economics from a macroperspective [21-23]. For 
example, Meepetchdee and Shah [24] discuss the growth, robustness, and benefits of logistics networks and uses the complex networks to simulate logistics networks. Viljoen and Joubert [25] uses complex networks to discuss stability. In addition, many scholars use the complex network theory to explore the structural characteristics of urban traffic networks and aviation networks [26-29]. However, the weights of network connections are not considered in the above studies. Some of the weights being chosen are unreasonable, which leads to a large gap between the established regional logistics network and the actual situation. Therefore, it is necessary to construct a more realistic regional logistics network and propose specific logistics network optimization strategies.

When studying the regional logistics network on a complex network, we usually simulate the regional logistics network based on theoretical knowledge by considering the interaction between nodes and the influence of the network on the nodes. In this paper, we establish the index of an urban logistics linkage based on the gravity model and the self-selection characteristics of logistics nodes. Then, the intercity logistics linkages are introduced into the regional logistics network as the connection weight, and the regional logistics weighted network is established. Such an investigation provides a new approach to construct the regional logistics weighted network model. On this basis, combined with complex network theory, the statistical properties and topological structure of the regional logistics weighted network are discussed, the stability and robustness of the network are analyzed, and a more stable topological structure model of the regional logistics network is found. Compared with the previous research, we use the failure area to describe the response of the regional logistics network under random attacks and deliberate attacks. The consideration is comprehensive. Then, we improve the comprehensive robustness optimization model, discuss how to maximize the comprehensive robustness of the regional logistics network at a limited cost, and search for the optimal solution through Python.

The reminder of the paper is organized as follows. In Section 2, we describe the mechanism of building a regional logistics weighted network model. In Section 3, we take Jiangsu-Zhejiang-Shanghai region, the most developed e-commerce region in China, as an example to establish a regional logistics weighted network. Then, we analyze the statistical characteristics of the system using complex network theory. In Section 4, we discuss the robustness of the regional logistics weighted network, develop the optimization strategy of the model and consider its robustness, and seek the optimal solution of the model at a limited cost. Conclusions are drawn in Section 5.

\section{Regional Logistics Weighted Network}

Regional logistics network is mainly composed of nodes and edges, in which nodes denote different cities and edges mainly represent transportation routes between cities. Since the focus of this paper is on the study of logistics in a small area, the nodes represent county-level cities within the region and the edges represent transportation roads.

We use $Z$ to denote all nodes $i$ in the regional logistics network, $N$ the node connection matrix, and $n(i, j)$ the connection between nodes $i$ and $j$. Thus, $N$ is given by

$$
N=\{(i, j) \mid n(i, j)=1, \quad(i, j) \in Z, n \in N\} .
$$

For $n(i, j)=1$, it implies that there is a logistics connection between node $i$ and node $j$, and $n(i, j)=0$ means that there is no logistics connection between node $i$ and node $j$. Based on formula (1), we establish a weighted regional logistics network model which has weighted information of all edges between nodes. Thus,

$$
W=\left\{(i, j) \mid w(i, j)=H_{i j}, \quad(i, j) \in Z, h \in U\right\},
$$

where $w(i, j)$ denotes the logistics connection weight between node $i$ and node $j$ and $U$ denotes the connection weight matrix between nodes. The construction method of $H_{i j}$ is described below.

According to the gravity model, the index of intercity logistics linkages is established and hence the weight index of regional logistics network. Based on the autonomous selectivity and preference of nodes in the regional logistics network and the joining of the regional logistics network, new nodes often establish logistic linkages with nodes having strong logistics competitiveness. From a market perspective, the supply-demand relationship of logistics industry directly reflects the logistics competitiveness of nodes, while the freight volume of the node directly reflects the demand of the node logistics industry. The logistics supply capacity of the node is determined by the logistics cost. The logistics cost mainly refers to the transportation cost and labor cost, which is proportional to distance. The greater the distance between two nodes, the greater the cost of forming a logistics connection. In summary, we select two indices from the logistics evaluation system: the freight volume of the node and the road distance between two nodes. Nodes with large freight volumes have high logistics demands and are more attractive to surrounding nodes. On the other hand, the shorter the distance between two nodes, the lower the cost of forming logistics connection. The regional logistics system is expressed as the abovementioned supply and demand model. Through the construction of the gravity model, we take the intercity logistics linkage index $R_{i j}$ as the influencing factors of the system, which is positively correlated with the freight volume and negatively correlated with the distance between two nodes. Thus,

$$
\begin{aligned}
& R_{i j}=\frac{\sqrt{M_{I}} * \sqrt{M_{j}}}{D_{i j}}, \\
& H_{i j}=\frac{R_{i j}}{\sum_{i=1}^{n} R_{i j}},
\end{aligned}
$$

where $M_{i}$ and $M_{j}$ are freight volumes of node $i$ and node $j$, respectively, $D_{i j}$ is the distance between $i$ and $j$, and $H_{i j}$ denotes the degree of node $i$ and node $j$. We use $H_{i j}$ to denote the weight between node $i$ and node $j$ in (2), i.e., 
$h=H_{i j}$. The regional logistics weighted network can be described as

$$
R=(Z, N, U),
$$

where $Z$ is the set of all nodes, $N$ is the connection state between nodes, and $U$ is the connection weight between nodes.

\section{Case Study: Regional Logistics Weighted Network in Jiangsu-Zhejiang- Shanghai Region}

Taking Jiangsu-Zhejiang-Shanghai region as an example, we establish a regional logistics weighted network. In this model, each node represents a city. The connection weight is the degree of logistics membership between nodes. We get the latest freight volume from the 2017 Statistical Yearbook of China. The shortest road distance between cities is obtained from the map of China. According to formula (3), we get the attraction of intercity logistics linkages among 118 nodes. After further processing, we get the membership degree of nodes and then construct the regional logistics weighted network in this area. The results of the regional logistics weighted network obtained by Ucinet are shown in Figure 1. As shown in the Figure 1, the cities with large freight volumes, such as Shanghai, Hangzhou, and Ningbo, are more attractive to the surrounding cities, and as such, it is easy for these cities to generate contacts with nodes. These cities are in network-intensive areas. The cities far away from the surrounding cities with small freight volume are difficult to generate contacts with the network. These cities are in the marginal area of the regional logistics network. The situation shown in Figure 1 is basically consistent with the reality. Based on the complex network theory, we analyze the network structure of the regional logistics weighted network from the statistical characteristics.

3.1. Degree and Degree Distribution. The degree of a node refers to the number of other nodes connected to the node, which is often used to describe the capacity of the node. We use $k_{i}$ to represent the degree of node $i, k_{i}=\sum_{i \neq j} n(i, j)$. The average degree $k$ of the network is 9.8136, and the degree distribution of the logistics network is shown in Figure 2. From Figure 2, we see that Suzhou is the node with the largest degree, up to 43 , followed by Wuxi (32), Shaoxing (30), Jiande (28), Hangzhou (21), Shanghai (19), and Nanjing (18). We note that although Shanghai's GDP is much higher than its surrounding cities such as Hangzhou, it is not the most important logistics node in the network. Therefore, we should not only consider regional GDP to select the central node of the regional logistics network. The location factor should also be taken into consideration as a reference index.

As shown in Figure 3, we find that only a few nodes in the regional logistics weighted network have high degree. Most nodes have degrees distributed between 0 and 15. The regional logistics weighted network is very uneven having the characteristics of scale-free network. This kind of network has obvious weaknesses. Attacks on key nodes in the network can easily lead to large-scale paralysis. On the other hand, the uneven structure of network model can easily cause network congestion. Based on the above discussion, since it is easy for the nodes in Suzhou, Wuxi, Shaoxing, Jiande, and Hangzhou to produce logistics links with their surrounding nodes, it is more reasonable, from the perspective of the construction of the whole regional logistics network, to choose these nodes as the central locations of the regional logistics network.

Through the above analysis on the degree distribution, we find that a small number of nodes in the regional logistics network are hub nodes, and the degrees of most of the nodes are relatively small. Furthermore, we measure the degree distribution characteristics of the regional logistics network by the cumulative distribution function $P>k$, as shown in Figure 4 . We assume that there is a constant $\mathrm{C}$ and a power exponent $\gamma$, which satisfy $P \sim \mathrm{Ck}^{-\gamma}$. Then, we can infer that the processed data approximately conform to the power-law distribution. We use SPSS for exponential fitting and use the nonparametric KolmogorovSmirnov test to verify the hypothesis. The results are shown in Tables 1 and 2. Therefore, we believe that the regional logistics weighted network has scale-free network characteristics and thus is a scale-free network.

3.2. Average Shortest Path Length. The shortest path length $L_{c}$ refers to the path connecting the two nodes with the least number of edges in the network. The average shortest path length $L$ is the average of the shortest path lengths of all nodes in the network. The average shortest path length $L$ of the regional logistics weighted network is 3.069. Although the structure of the network seems complex and huge, the distance between nodes is not far away, which further shows that the network has the characteristics of small world network according to complex network theory.

3.3. Betweenness Centrality. Betweenness centrality is an index to describe the importance of a node by the number of the shortest paths of the node. The distribution of the betweenness centrality of the regional logistics weighted network is shown in Figure 5, which shows that there are five distinct peaks corresponding to Jiande, Dongyang, Changzhou, Suzhou, and Yizheng. The above nodes have significant influences on the connectivity of the network. In addition, we also find that the nodes of Yongjia, Pingyang, Pujiang, Kaihua, Daishan, Qixian, Xianju, Longquan, Qingyuan, Suichang, Jingning, Pei, Kunshan, Taicang, Qidong, Jurong, Jingjiang, Taixing, Shuyang, and Sihong have betweenness centrality 0 . These nodes contribute little to network connectivity, and hence they can almost be ignored.

3.4. Clustering Coefficient. The clustering coefficient $C_{i}$ refers to the connection between other nodes connected to $i$. The higher the clustering coefficient $C_{i}$ is, the closer the relationship between the neighbors of $i$ is. The clustering coefficient $C_{i}$ is defined as 


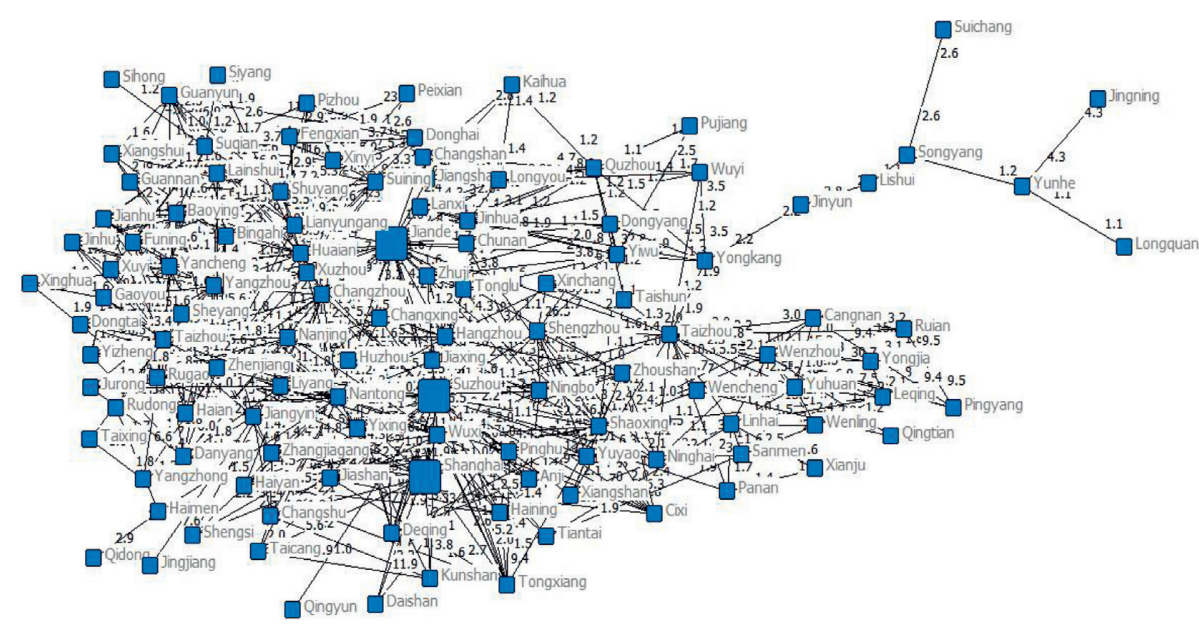

FIgURE 1: The regional logistics weighted network of Jiangsu-Zhejiang-Shanghai area.

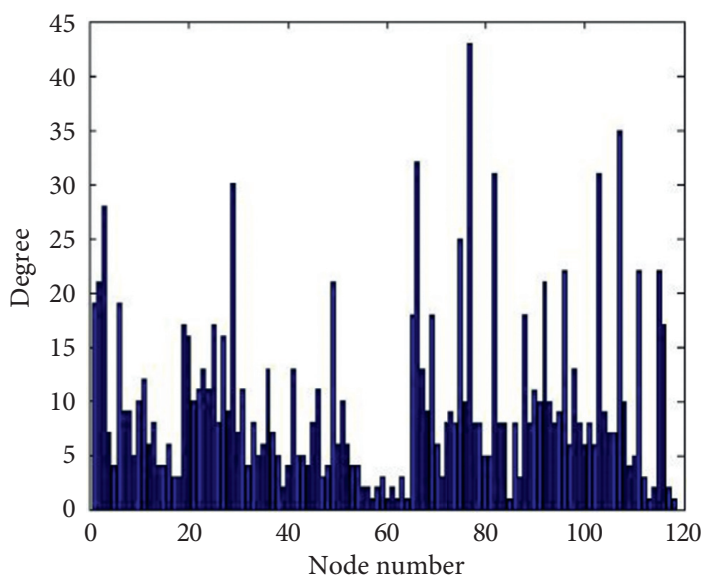

Figure 2: The degree of nodes.

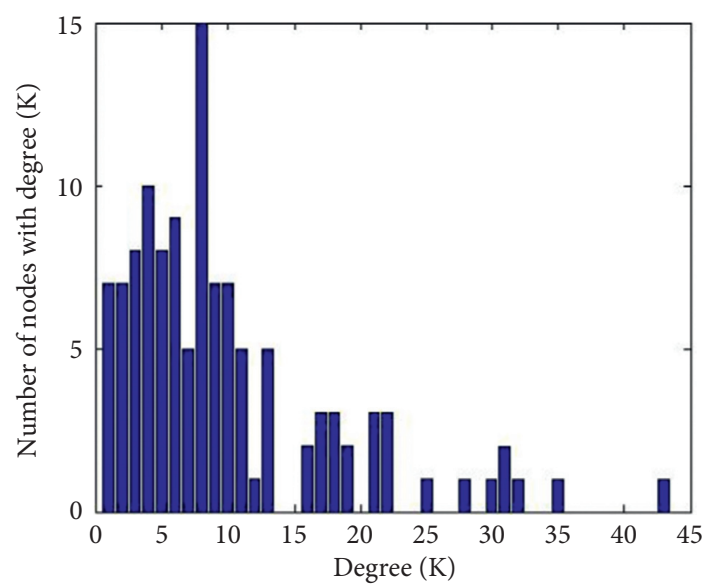

FIgURE 3: The degree distribution.

$$
C_{i}=\frac{2 E_{i}}{k_{i}\left(k_{i}-1\right)}
$$

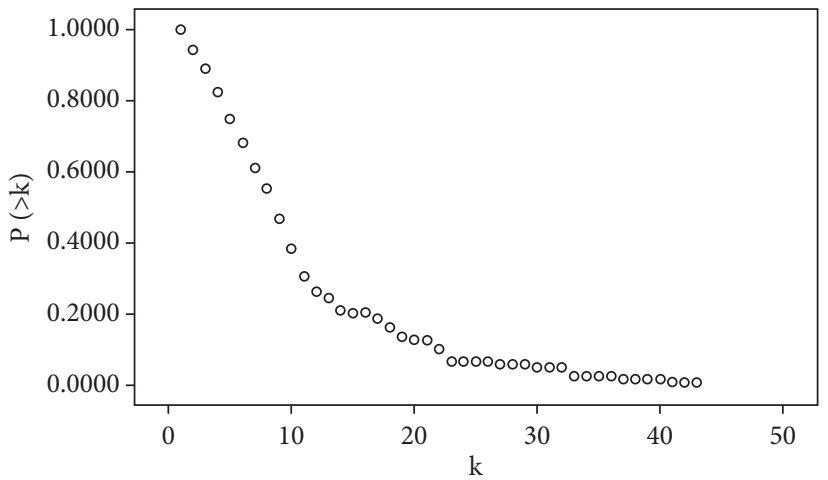

Figure 4: The cumulative distribution.

The average clustering coefficient $C$ refers to the average of the clustering coefficients of all nodes in the network. The average clustering coefficient $C$ of the regional logistics weighted network of Jiangsu-Zhejiang-Shanghai region is obtained as 0.5868 using MATLAB. There are many nodes in the regional logistics weighted network, these nodes are actually closely linked through the connection, and the distance between nodes is much smaller than imagined. The clustering coefficients of each node are shown in Figure 6.

According to Figures 1 and 6, the clustering coefficients of Yongjia, Pingyang, Pujiang, Kaihua, Daishan, Shengsi, Xianju, Peixian, Kunshan, Taicang, Jurong, Taixing, and Siyang counties are 1 , which indicate that the other nodes connected with these cities are fully connected. The above nodes are in a very stable network structure. The clustering coefficients of Wencheng, Taishun, Lishui, Longquan, Qingtian, Yunhe, Qingyun, Jinyun, Suichang, Songyang, Jingning, Qidong, Jingjiang, and Sihong are 0, implying that these nodes are totally disconnected. If such nodes are in the bridge position in the network model, they will easily cause network paralysis when attacked. Figure 1 shows that this kind of nodes either have only one connection node, or the nodes connected to them are disconnected. If such nodes are in the bridge position in the network, they will easily cause network paralysis when attacked. 
TABLE 1: Summary of statistical analysis.

\begin{tabular}{|c|c|c|c|c|c|}
\hline \multicolumn{6}{|c|}{ Coefficient } \\
\hline & \multicolumn{2}{|c|}{ Coefficient of nonstandardization } & \multirow{2}{*}{$\begin{array}{c}\text { Standard coefficient } \\
\beta\end{array}$} & \multirow{2}{*}{$t$} & \multirow{2}{*}{ Significance } \\
\hline & $B$ & Standard error & & & \\
\hline Degree & -0.112 & 0.002 & -0.994 & -57.093 & 0.000 \\
\hline (Constant) & 1.205 & 0.060 & & 20.181 & 0.000 \\
\hline
\end{tabular}

TABLE 2: Summary of hypothesis tests.

\begin{tabular}{lccc}
\hline Original hypothesis & Test & Significance & Decision \\
\hline $\begin{array}{l}\text { The cumulative distribution is exponential distribution with } \\
\text { an average of } 0.200\end{array}$ & $\begin{array}{c}\text { Single-sample } \\
\text { KolmogorovSmirnov test }\end{array}$ & 0.062 & Keep the original hypothesis \\
\hline
\end{tabular}

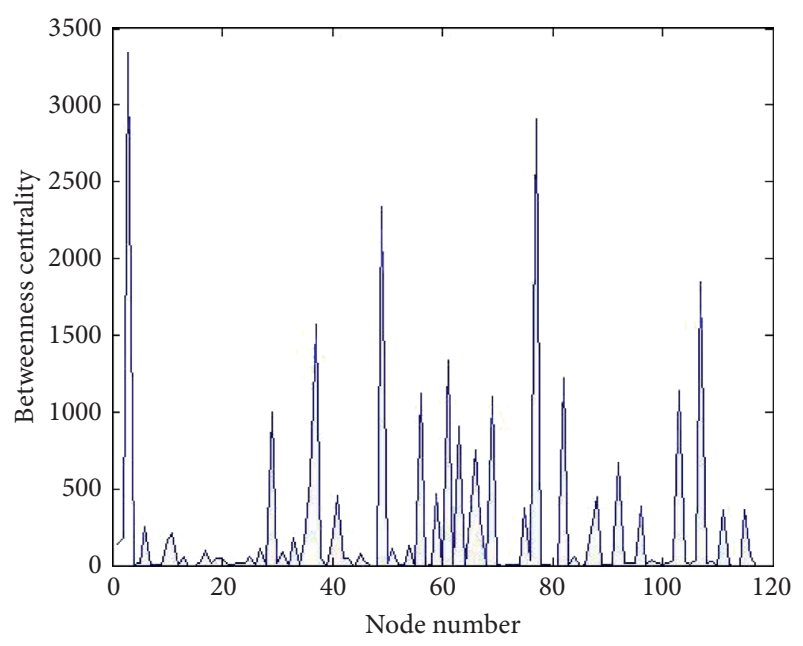

FIgURE 5: Betweenness centrality.

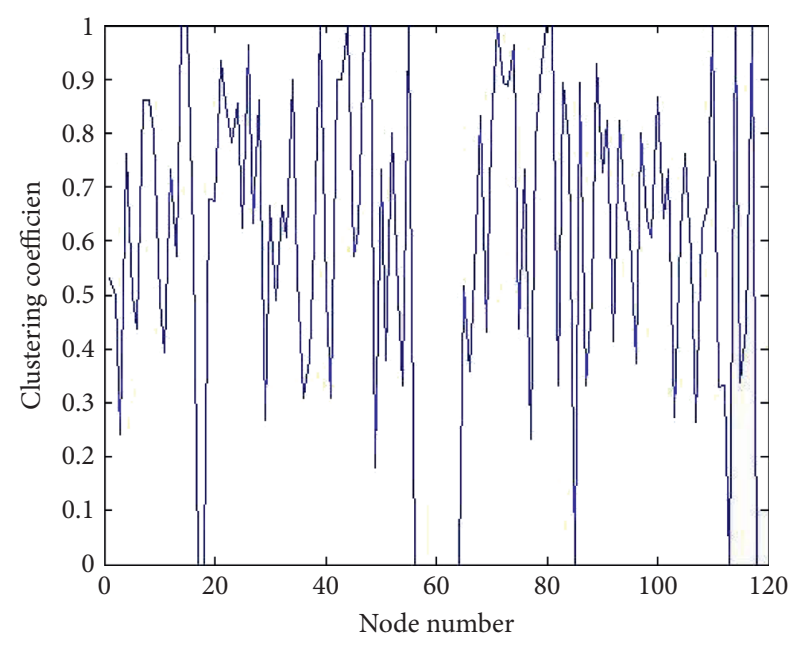

Figure 6: Clustering coefficient.

\section{Robustness of Regional Logistics Weighted Network}

The regional logistics network is the basis of interregional economic cooperation and an important guarantee for the coordinated development of regional economies. Through the above analysis of the statistical characteristics of the regional logistics weighted network, it is found that the network has an extremely uneven network structure. For this kind of network structure, the failure of logistics nodes or edges caused by traffic jams, traffic accidents, natural disasters, bad weather, or major incidents may lead to largescale failure of the logistics network. Therefore, it is of great significance to discuss how to improve the ability of the regional logistics network to respond to various emergencies. We further analyze the robustness of regional logistics network model and discuss methods to optimize the robustness of the network at a limited cost.

4.1. Robustness Analysis of the Network. The robustness analysis of a complex network refers to the analysis of the connection state between nodes and the change of the whole network connection state when the network is attacked intentionally or randomly. Intentional attack refers to the failure of a certain percentage of important nodes or edges in the network. Random attack refers to completely random failures of nodes or edges in the network. In this paper, we choose the relative scale of the maximum connected subgraph $Q$ to measure the robustness of the network after being attacked.

$$
Q=\frac{s}{S}
$$

where $s$ is the maximum connected subgraph range of the network when the network is attacked and $S$ is the initial scale of the network. The smaller the relative scale of the maximum connected subgraph, the more serious the damage of the regional logistics network is. Simulate the above situation by Python, the results are shown in Figures 7 and 8 . Figure 7 shows the trend of $Q$ under various node failure strategies. Figure 8 shows the trend of $Q$ under various edge failure strategies. From Figure 7, we can see that in the case of random attack on nodes in the regional logistics weighted network, $Q$ shows a linear trend and slowly decreases as the number of nodes being attacked increases. Under random attack, the effect of each node being attacked is not much different, so it will only reduce $Q$ by a very small value. This clearly shows that the regional logistics weighted 


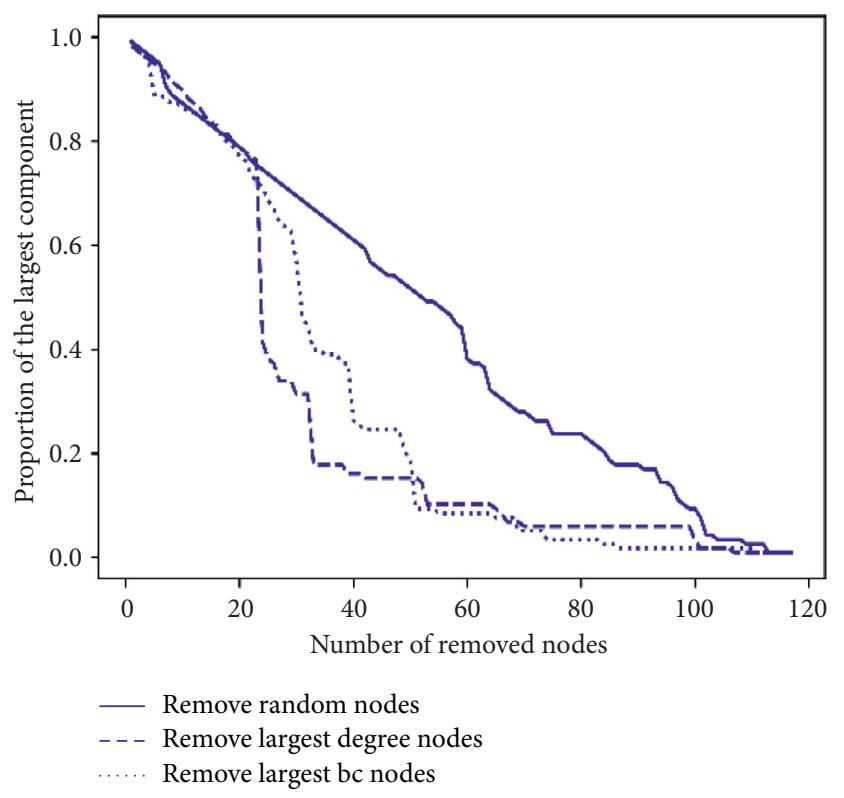

FIgURE 7: Robustness analysis under node failure.

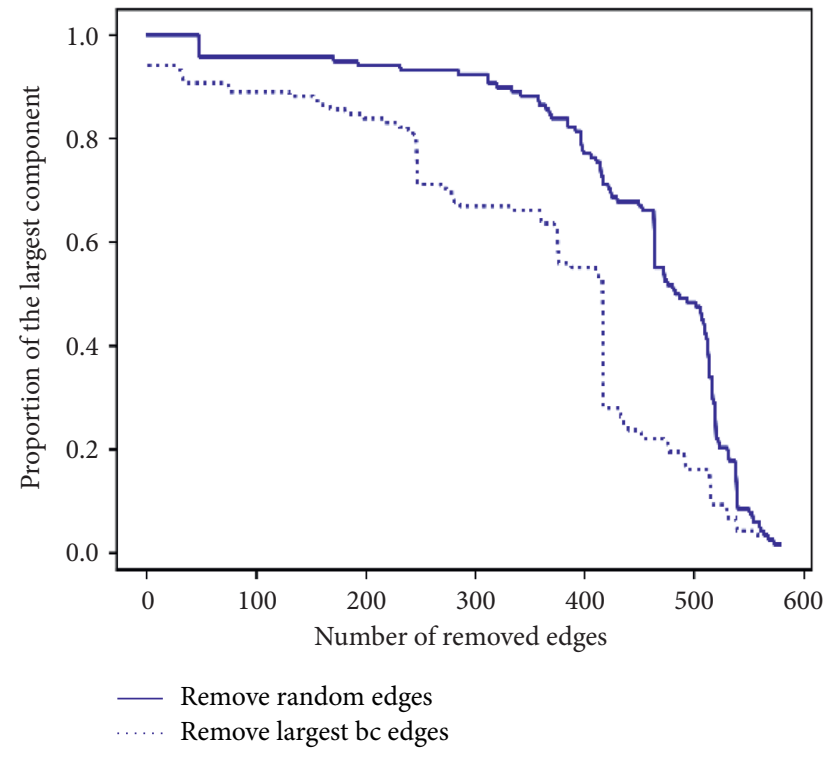

Figure 8: Robustness analysis under edge failure.

network has strong robustness under random attack. Under intentional attacks, $Q$ decreases as the number of failed nodes increases. When the number of failed nodes reaches 20 , the relative scale of the maximum connected subgraph $Q$ of the regional logistics weighted network shows a rapid shrinking trend, indicating that the regional logistics weighted network is no longer robust. This phenomenon is consistent with the characteristics of scale-free networks, which is robust to random failures but is vulnerable to intentional attacks. Figure 8 shows that under random attack, when the number of failed edges of the regional logistics weighted network is less than 300 , the $Q$ value of the network will not change significantly and basically remains at about 0.95 . Under intentional attacks, the robustness of the network is obviously weaker than that under random attacks. When the number of failed edges in the network exceeds 100, the decline rate of the $Q$ value increases significantly. When the number of failed edges exceeds 200, the $Q$ value will decrease linearly and the regional logistics weighted network will be paralyzed in a large area. The regional logistics weighted network is not very robust to the intentional attack strategy of nodes or edges. Therefore, we propose a robust optimization model based on the existing robustness research and discuss how to maximize the robustness of the regional logistics weighted networks under a limited cost.

4.2. Robustness Optimization of the Network. At present, there are few studies on the robust optimization of scale-free networks. In [30, 31], an in-depth study of the phase transition characteristics of the scale-free networks is carried out, where criteria for determining the existence of the largest interconnected group in the network are derived, and the threshold $P^{c}$ is established for scale-free networks in response to intentional and random attacks. When the onetime failure ratio of scale-free network $P>P^{c}$, scale-free network will fall into a large area of failure $[30,31]$. On this basis, by combining the penetration theory and an optimization method, an optimization model of scale-free networks under random and intentional attacks is proposed in [32]. More specifically, based on the percolation theory and an optimization method, it can quantify the robustness index of the regional logistics network in response to random attacks and intentional attacks, Then, it will be able to establish a comprehensive robust optimization model of the regional logistics system under the cost constraints and to find the optimal solution of the robust optimization model under a limited cost.

The aim of this paper is to maximize the threshold $P^{c}$ of the network subject to the constraints on the cost. When scale-free networks are attacked randomly, the threshold $P_{\text {random }}^{c}$ is

$$
P_{\text {random }}^{c}=1-\frac{1}{k_{0}-1},
$$

where $k_{0}$ can be obtained as

$$
k_{0}=\frac{\sum_{k=m}^{K} k^{2}}{\sum_{k=m}^{K} k},
$$

while $m$ is the minimum node degree and $K$ is the maximum node degree. When scale-free network is attacked intentionally, the threshold $P_{\text {target }}^{c}$ is given by

$$
P_{\text {target }}^{c}=\left(\frac{K^{*}}{m}\right)^{1-\gamma}-\frac{1}{n},
$$

where $n$ is the number of network nodes, $K^{*}$ is the maximum node degree after the node is inactivated, and $\gamma$ is the exponential value of the degree distribution of the network. Here, $K^{*}$ is given by 


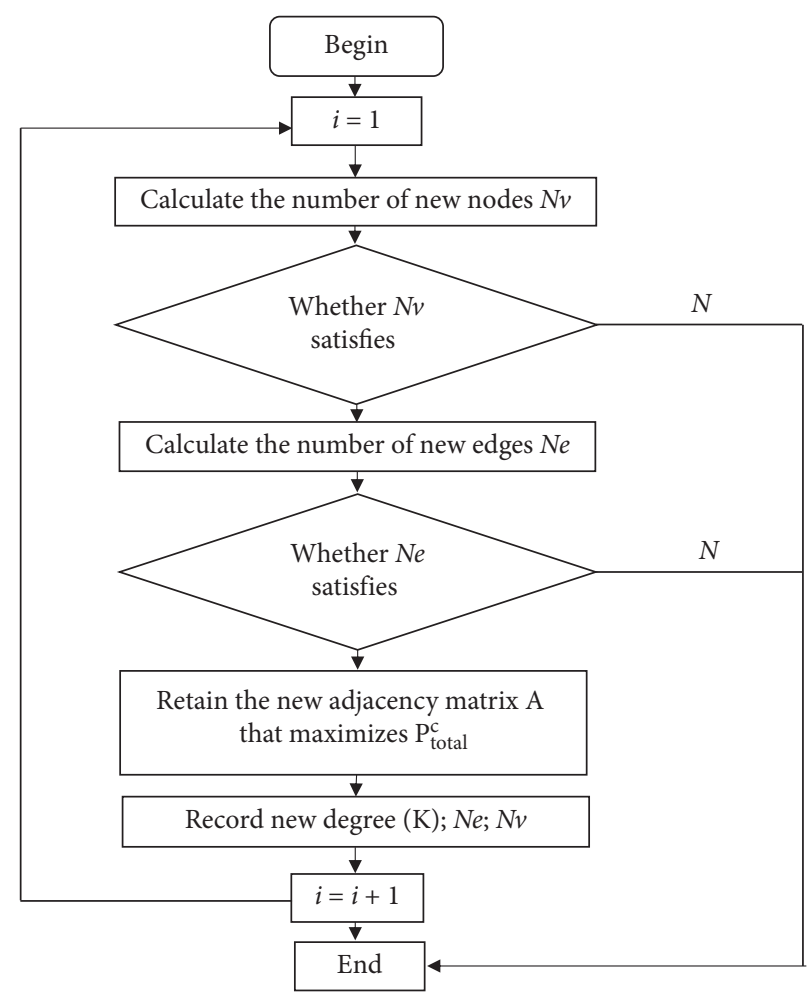

FIgURE 9: Flow chart of robust optimization.

TABLE 3: Regional logistics network optimization results.

\begin{tabular}{lccc}
\hline (Chunan, Pujiang) & (Tonglu, Tongxiang) & (Tonglu, Zhenjiang) & (Tonglu, Leqing) \\
\hline (Chunan, Deqing) & (Shanghai, Jinhua) & (Chunan, Longyou) & (Yuyao, Wenchen) \\
\hline (Ruian, Shaoxing) & (Shanghai, Yiwu) & (Yuyao, Zhenjiang) & (Cixi, Suining) \\
\hline (Yongjia, Cangnan) & (Xiangshan, Zhenjiang) & (Shaoxing, Nantong) & (Shaoxing, Yangzhou) \\
\hline (Shaoxing, Zhenjiang) & (Jiande, Shaoxing) & (Hnagzhou, Zhenjiang) & (Shaoxing, Changzhou) \\
\hline
\end{tabular}

$$
\left(\frac{K^{*}}{m}\right)^{2-\alpha}-2=\frac{2-\alpha}{3-\alpha} m\left[\left(\frac{K^{*}}{m}\right)^{3-\alpha}-1\right] .
$$

Therefore, the comprehensive threshold of robustness can be expressed as

$$
P_{\text {total }}^{c}=\alpha P_{\text {random }}^{c}+\beta P_{\text {target }}^{c},
$$

where $\alpha$ and $\beta=(1-\alpha)$ are weights of random attack and intentional attack thresholds, respectively. It is known that the robustness of the logistics network will be enhanced when new nodes or connections are introduced. However, the cost of establishing new logistics nodes or links is high. Based on the above theory, we design the robust optimization model for the regional logistics network under fixed cost.

To enhance the robustness of regional logistics network, new logistics warehouses can be added between current logistics nodes and new logistics links can be established between nodes. We assume that the total cost is $C$, the cost of introducing a new logistics node is $a$, and the cost of establishing a new logistics connection is $b$. The robust optimization model of the regional logistics network can be expressed as

$$
\begin{gathered}
\max P_{\text {total }}^{c}=\alpha P_{\text {random }}^{c}+\beta P_{\text {target }}^{c} \\
\text { s.t.: } \quad a_{V}+\mathrm{bN}_{e} \leq \mathrm{C} \\
0 \leq N_{v}<\frac{C}{a} \\
0<N_{e} \leq \frac{C}{b},
\end{gathered}
$$

where $N_{V}$ is the number of newly introduced nodes and $N_{e}$ is the number of newly established logistics connections. The design procedures of the optimization program are as follows:

(i) Introduce new nodes or edges to the regional logistics network. Then, test whether or not the cost constraints in the optimization model are met. 
(ii) If the restrictions are not met, the cost has been used up and the result is obtained. If the constraints are satisfied, calculate the $P_{\text {total }}^{c}$ of the regional logistics network with the new nodes or edges having introduced to all its possible locations. The maximum value of $P_{\text {total }}^{c}$ is retained.

(iii) Record the location of the newly introduced nodes and edges, output $P_{\text {total }}^{c}$, and the new connection matrix.

The logic diagram of the optimization program is shown in Figure 9, which is implemented by Python.

For the regional logistics weighted networks, the ability to resist intentional attacks is more important than the ability to resist random attacks. Therefore, we assume that the weight of random attack $\alpha=0.3$ and the weight of intentional attack robustness $\beta=0.7$. The $P_{\text {random }}^{c}$ of the initial logistics network is 0.93522 , the $P_{\text {target }}^{c}$ of the initial logistics network is 0.001162 , and the comprehensive threshold $P_{\text {total }}^{c}$ is 0.281379 . Assume that the cost of establishing new logistics nodes $N_{V}$ is 20 , the cost of establishing new logistics connections $N_{e}$ is 10 , and the total cost $C$ is 200 . Through the above optimization program, the output result is $N_{V}=0$ and $N_{e}=20$; the locations of including new nodes and connections are listed in Table 1 . The $P_{\text {random }}^{c}$ of the optimized regional network is 0.93792 , and the $P_{\text {target }}^{c}$ of the optimized regional network is 0.041338 . The comprehensive robustness threshold $P_{\text {total }}^{c}=0.31031$ is $8.8 \%$ higher than that of the original network robustness.

Based on the above discussion, we believe that when we optimize the robustness of the regional logistics weighted network of Jiangsu-Zhejiang-Shanghai region under a limited cost, adding the above connections as shown in Table 3 can maximize the comprehensive robustness of regional logistics.

\section{Conclusion}

In this work, our study was mainly focused on the construction of regional logistics network and the development of robust optimization. We established the regional logistics weighted network from a macroperspective. Through the discussion and analysis of this paper, the summary is as follows:

(1) Compared with previous studies from the single node location [2-5] or hub model [14], we studied the logistics activities of a region from the perspective of macronetwork. According to the relevant economic research, we believe that the logistics nodes have self-selection capabilities and constructed the attractiveness index of logistics nodes as the weight of the regional logistics network. It is found that the economically developed cities are located in dense areas of logistics networks. For economically underdeveloped cities, they are located in sparse areas of logistics network. The results obtained are in line with the actual situation. This also verifies the feasibility of our modeling ideas.
(2) We analyze the regional logistics network model of Jiangsu-Zhejiang-Shanghai through the social network method. The average shortest path length is 3.069 and the aggregation coefficient is 0.5868 . Although there are many nodes in a single regional logistics weighted network model, the average degree and average path length are not large, which shows that it has obvious small world characteristics. On this basis, we can provide more economic insights for the construction of regional logistics networks.

(3) Through robustness analysis, it is found that the Jiangsu-Zhejiang-Shanghai region logistics network model is robust in the face of random attacks and is prone to large-scale failures when facing deliberate attacks. This is also a common feature of scale-free networks. When $\alpha=1$ and $\beta=0$, the robust optimization model can resist random attacks which is in line with the results obtained in [28]. When $\alpha=0$ and $\beta=1$, the robust optimization model can resist deliberate attacks which agrees with the results reported in [29]. We comprehensively considered the response of the regional logistics network to the two kinds of attacks. Then, we established a comprehensive robustness index for the critical value of large-area failures and discussed how to maximize performance under fixed costs. Finally, taking Jiangsu-Zhejiang-Shanghai region logistics network model as an example, specific experimental results are given.

When establishing a robust optimization model, this paper simplifies the actual situation by ignoring the difference in connection costs between different nodes. We only discuss static models. In future research, various practical factors should be taken into account in the investigation of the regional logistics networks.

\section{Data Availability}

Some or all data, models, or code generated or used during the study are available from the corresponding author by request.

\section{Conflicts of Interest}

The authors declare that there are no conflicts of interest regarding the publication of this paper.

\section{Acknowledgments}

This work was partially supported by the National Natural Science Foundation of China (Nos. 71501069, 71420107027, and 71871030), Humanities and Social Sciences Project of the Ministry of Education of China (No. 19YJAZH132), Scientific Research Project of Hunan Provincial Education Department in China (No.18A037), Hunan Key Laboratory of Macroeconomic Big Data Mining and its Application, and a Discovery Grant from Australian Research Council. 


\section{References}

[1] C. S. Revelle and R. W. Swain, "Central facilities location," Geographical Analysis, vol. 2, no. 1, pp. 30-42, 2010.

[2] R. Tadei, N. Ricciardi, and G. Perboli, "The stochastic -median problem with unknown cost probability distribution," $O p$ erations Research Letters, vol. 37, no. 2, pp. 135-141, 2009.

[3] S. Elloumi, "A tighter formulation of the $p$-median problem," Journal of Combinatorial Optimization, vol. 19, no. 1, pp. 69-83, 2010.

[4] Y. An, B. Zeng, Y. Zhang, and L. Zhao, "Reliable p-median facility location problem: two-stage robust models and algorithms," Transportation Research Part B: Methodological, vol. 64, pp. 54-72, 2014.

[5] M. Albareda-Sambola, Y. Hinojosa, and J. Puerto, "The reliable $\mathrm{p}$-median problem with at-facility service," European Journal of Operational Research, vol. 245, no. 3, pp. 656-666, 2015.

[6] A. Saxena, V. Goyal, and M. A. Lejeune, "MIP reformulations of the probabilistic set covering problem," Mathematical Programming, vol. 121, no. 1, pp. 1-31, 2010.

[7] O. Baron, O. Berman, S. Kim, and D. Krass, "Ensuring feasibility in location problems with stochastic demands and congestion," IIE Transactions, vol. 41, no. 5, pp. 467-481, 2009.

[8] O. Berman and J. Wang, "The minmax regret gradual covering location problem on a network with incomplete information of demand weights," European Journal of Operational Research, vol. 208, no. 3, pp. 233-238, 2011.

[9] G. Alexandris and I. Giannikos, "A new model for maximal coverage exploiting GIS capabilities," European Journal of Operational Research, vol. 202, no. 2, pp. 328-338, 2010.

[10] L. Yan, F. Xu, J. Liu, K. L. Teo, and M. Lai, "Stability strategies of demand-driven supply networks with transportation delay," Applied Mathematical Modelling, vol. 76, pp. 109-121, 2019.

[11] S. Xie, X. Li, and Y. Ouyang, "Decomposition of general facility disruption correlations via augmentation of virtual supporting stations," Transportation Research Part B: Methodological, vol. 80, pp. 64-81, 2015.

[12] L. Yan, J. Liu, and Z. Luo, "Existence and multiplicity of solutions for second-order impulsive differential equations on the half-line," Advances in Difference Equations, vol. 63, pp. 1-12, 2013.

[13] Z. Yu-Feng, M. A. Zu-Jun, and W. Ke-Ming, "Reliability capacitated fixed-charge location problem," Operations Research and Management Science, vol. 11, pp. 677-686, 2015.

[14] C. S. Sung and H. W. Jin, "Dual-based approach for a hub network design problem under non-restrictive policy," $\mathrm{Eu}$ ropean Journal of Operational Research, vol. 132, no. 1, pp. 88-105, 2001.

[15] R. Ishfaq and C. R. Sox, "Hub location-allocation in intermodal logistic networks," European Journal of Operational Research, vol. 210, no. 2, pp. 213-230, 2011.

[16] S. A. Alumur, B. Y. Kara, and O. E. Karasan, "Multimodal hub location and hub network design," Omega, vol. 40, no. 6, pp. 927-939, 2012.

[17] J. T. Bowen, "A spatial analysis of FedEx and UPS: hubs, spokes, and network structure," Journal of Transport Geography, vol. 24, pp. 419-431, 2012.

[18] N. Gallego, C. Llano, T. De La Mata, and J. DÍaz-Lanchas, "Intranational home bias in the presence of wholesalers, hubspoke structures and multimodal transport deliveries," Spatial Economic Analysis, vol. 10, no. 3, pp. 369-399, 2015.
[19] Y. Wang and R. Xiao, "An ant colony based resilience approach to cascading failures in cluster supply network," Physica A: Statistical Mechanics and Its Applications, vol. 462, pp. 150-166, 2016.

[20] L. Hu, J. X. Zhu, Y. Wang and L. H. Lee, Joint design of fleet size, hub locations, and hub capacities for third-party logistics networks with road congestion constraints," Transportation Research Part E: Logistics and Transportation Review, vol. 118, no. 10, pp. 568-588, 2018.

[21] T. Broekel, "The Co-evolution of proximities - a network level study," Regional Studies, vol. 49, no. 6, pp. 921-935, 2015.

[22] B. Derudder and P. J. Taylor, "Central flow theory: comparative connectivities in the world-city network," Regional Studies, vol. 52, no. 2, pp. 1-14, 2017.

[23] B. D. Vos, B. Raa, and S. D. Vuyst, "A savings analysis of horizontal collaboration among VMI suppliers," Journal of Industrial and Management Optimization, vol. 13, no. 5, pp. 1-19, 2017.

[24] Y. Meepetchdee and N. Shah, "Logistical network design with robustness and complexity considerations," International Journal of Physical Distribution \& Logistics Management, vol. 37, no. 3, pp. 201-222, 2007.

[25] N. M. Viljoen and J. W. Joubert, "The vulnerability of the global container shipping network to targeted link disruption," Physica A: Statistical Mechanics and Its Applications, vol. 16, pp. 785-792, 2016.

[26] J. Liu, Y. Huang, and C.-P. Chang, "Leverage analysis of carbon market price fluctuation in China," Journal of Cleaner Production, vol. 245, p. 118557, 2020.

[27] J. Liu, C. Cheng, X. Yang, L. Yan, and Y. Lai, “Analysis of the efficiency of Hong Kong REITs market based on Hurst exponent," Physica A: Statistical Mechanics and Its Applications, vol. 534, p. 122035, 2019.

[28] A. T. Buba and L. S. Lee, "Differential evolution with improved sub-route reversal repair mechanism for multiobjective urban transit routing problem," Numerical Algebra, vol. 8, no. 3, pp. 361-386, 2018.

[29] M. E. O'Kelly, "Global airline networks: comparative nodal access measures," Spatial Economic Analysis, vol. 11, no. 3, pp. 253-275, 2016.

[30] R. Cohen, K. Erez, D. Benavraham et al., "Breakdown of the internet under intentional attack," Physical Review Letters, vol. 86 , no. 16 , pp. 36-82, 2001.

[31] G. Paul, T. Tanizawa, S. Havlin, and H. E. Stanley, "Optimization of robustness of complex networks," The European Physical Journal B, vol. 38, no. 2, pp. 187-191, 2004.

[32] J. G. Liu, Z. T. Wang, and Y. Z. Dang, "Optimization of robustion of scale-free network to random and targeted attacks," Modern Physics Letters B, vol. 19, no. 16, pp. 785-792, 2008. 